\title{
A Subgroup of Patients with Chronic Fatigue Syndrome May Have a Disorder of Arousal
}

\author{
Toru Nakamura ${ }^{1}$, Fumiharu Togo ${ }^{3}$, Neil S. Cherniack ${ }^{2, \dagger}$, David Rapoport ${ }^{4}$ and Benjamin H. Natelson ${ }^{*, 1,5}$
}

\author{
${ }^{1}$ Departments of Neurosciences; ${ }^{2}$ Medicine, UMD-New Jersey Medical School, Newark; ${ }^{3}$ Department of Work Stress \\ Control, Japan National Institute of Occupational Safety and Health, Kawasaki, Japan; ${ }^{4}$ Department of Medicine, Divi- \\ sion of Pulmonary and Critical Care Medicine, NYU School of Medicine, New York, NY, USA; ${ }^{5}$ Pain \& Fatigue Study \\ Center, Department of Pain Medicine and Palliative Care, Beth Israel Medical Center, New York, NY, USA
}

\begin{abstract}
Objective: Patients with chronic fatigue syndrome complain of unrefreshing sleep, and some have poor sleep indicative of sleep disruption. We hypothesized that some CFS patients had a disorder of arousal which interfered with normal sleep which would manifest itself by difficulty falling asleep after a night of sleep deprivation. To test this, we determined sleep latency after a night of sleep deprivation.

Methods: Patients and healthy controls, previously habituated to sleeping in a sleep lab, returned for a normal night of sleep and for a night of sleep deprivation followed by determination of latency to fall asleep. Patients also had indwelling catheters to allow repeated sampling of plasma for cortisol, a hormone whose levels might reflect arousal.

Results: Five of 15 patients showed delays in falling asleep, relative to the healthy controls. Sleep latency following sleep deprivation correlated inversely with sleep efficiency on the normal sleep night. No significant difference was found in cortisol patterns across time for patients and controls on the sleep deprivation night. However, the slope of the curve was shifted up significantly for patients but not for controls on the normal sleep night.

Conclusion: A subgroup of patients has difficulty falling asleep after a night of sleep deprivation. These patients may have a disorder of arousal that interferes with their having normal sleep. CFS patients as a group show a shift upwards in the temporal pattern of cortisol during the night - a possible measure of their being stressed while sleeping.
\end{abstract}

Keywords: Chronic fatigue syndrome, sleep deprivation, arousal.

\section{INTRODUCTION}

Chronic fatigue syndrome is a medically unexplained illness characterized by fatigue impacting significantly on daily activity and accompanied by neuropsychiatric symptoms as well as others that resemble infectious and rheumatic diseases. One of the most prominent symptoms is unrefreshing sleep, a symptom which patients rate as the most severe of all [1]. In addition to patient self report, an early study made the point that a substantial number of CFS patients have a diagnosable sleep disorder - usually sleep apnea or restless leg syndrome [2]. However, even when such cases are excluded, other studies have noted subtle abnormalities including reduced sleep efficiency [3, 4] and differences in the microstructure of sleep as determined by power spectrum analysis of sleep EEG [5].

We have recently reported the sleep architecture of a sample of CFS patients after excluding patients with diagnosable sleep disorders and co-existing major depressive disorder. These patients differed significantly from matched controls in showing evidence of sleep disruption in the form of shorter sleep bouts and lower sleep efficiencies [6].

*Address correspondence to this author at the Pain \& Fatigue Study Center, Department of Pain Medicine and Palliative Care, Beth Israel Medical Center, 10 Union Square East, New York, NY, 10003, USA; Tel: 212-8446768; Fax: 212-844-8930; E-mail: bnatelson@bethisraelny.org

'deceased.
Further investigation found the patient group to be heterogene- ous with a subgroup of patients reporting that they felt sleep-ier after a night of sleep than before it; this group was the one with the lowest sleep efficiencies.

These facts led us to the hypothesis that some CFS patients might have a disorder of arousal. To test this, we determined the time to fall asleep after a night of sleep deprivation and measured plasma cortisol, a hormone known to reflect arousal [7], concurrently. If an underlying sleep disorder exists that is responsible for disrupted sleep, we expected that some patients would have a substantial delay in falling asleep. The data reported here supported that hypothesis. We also sought to determine if data from a normal night in the sleep lab might predict those patients who had difficulty falling asleep after a night of sleep deprivation.

\section{METHODS}

The subjects were women -15 with CFS and 12 healthy controls - ranging in age from 25 to 55 years old. Subjects with CFS were either physician referred or self referred in response to media reports about our research. Healthy controls were acquaintances of patients or responded to recruitment flyers, reported being in good health, and had a negative medical history and physical examination. No subject was taking antidepressants, opiates, steroids, hypnotics and other sedatives including benzodiazepines at the time of study. Patients fulfilled the 1994 case definition for CFS [8] 
and thus had no medical explanation for their symptoms based on history, physical examination or lab tests. Patients were further characterized as having severe CFS or not using previously published criteria [9] as well as for the presence of co-existing fibromyalgia (FM) based on the American College of Rheumatology's 1990 criteria [10] to see if either illness severity or presence of FM impacted on results. Psychiatric diagnosis according to DSM-IV criteria was made using the computerized version of the Diagnostic Interview Schedule (DIS-IV) [11]. The psychiatric interview was used to identify and then exclude from study the following DSMIV-based disorders: schizophrenia, eating disorders, substance abuse, or bipolar disorder as well as current major depressive disorder, a psychiatric disorder that can disrupt sleep [12]. Therefore patients and controls were all negative for these exclusionary psychiatric diagnoses. All subjects provided informed consent, approved by the medical school's Institutional Review Board to participate in this research.

Subjects spent an initial night in the sleep laboratory to habituate them to sleeping while instrumented and to diagnose sleep apnea, narcolepsy or periodic leg movement disorder - the existence of which served to exclude them from subsequent research [6]; thus, every subject in this study was negative. On the day prior to this and subsequent studies, subjects were instructed to refrain from alcohol and caffeine ingestion and avoid engaging in prolonged and/or strenuous exercise except when otherwise instructed. Within 6 months of the habituation study, subjects returned to the sleep lab during the follicular phase of their menstrual cycles for a normal sleep night (complete data available elsewhere [13]); at approximately $10 \mathrm{pm}$ on that night, a venous catheter was inserted and instrumentation was put in place to allow recording of electroencephalogram (EEG; C3/A2, O1/A2 and FZ/A2), electrooculogram (EOG), submental electromyogram (EMG), and a lead II electrocardiogram (ECG).

Immediately after undergoing instrumentation, subjects completed visual analog scales on the state of their fatigue and sleepiness at that point in time. Subjects then went to sleep, and blood was sampled remotely for serum cortisol without disturbing the subjects via an extension catheter to another room three times while they slept (i.e., at $1 \mathrm{am}, 3 \mathrm{am}$ and $5 \mathrm{am}$ ). The next morning, subjects again completed another set of visual analog scales. The sleep record was scored by a single scorer according to standard criteria of Rechtschaffen and Kales every 30 s [14].

Subjects subsequently returned to the sleep lab during the follicular phase of their menstrual cycles and underwent the same instrumentation. However, they remained awake thereafter throughout the night, interacting as needed with the sleep nurse to assure a wakeful state. Blood was drawn on the same schedule as on the normal sleep night. On the next morning, subjects then completed another set of visual ana$\log$ scales and then were allowed to go to sleep in a darkened, quiet room at about $8 \mathrm{am}$. Sleep onset was defined as the first three consecutive epochs of sleep Stage 1 or the first epoch of other stages of sleep.

Cortisol was measured by radioimmunoassay (RIA) using an immune-coated tube cortisol RIA kit obtained from MP Biomedicals, LLC (Orangeburg, NY) with a sensitivity of $0.5 \mu \mathrm{g} / \mathrm{dl}$. The intraassay coefficient of variation was
$1.85 \%$ and the interassay coefficient of variation was $10.91 \%$.

\section{STATISTICAL METHODS}

We compared sleep latencies between patient and control groups with a $t$-test following square root transformation of the data because of heterogeneity of variance. We also examined the group differences in the sleep indices using one-way ANOVA for three group comparisons, and Mann-Whitney $U$-test for pair-wise comparisons. For the cortisol data, we used a $t$-test to determine baseline differences (cortisol levels at am 1:00) between CFS and Control groups; these did not exist. Then, to deal with missing cortisol data $(<0.1 \%$ of total data points), we used a linear mixed model for subsequent analysis. In the model, we treated group and sampling times (at 1:00 am, 3:00 am, and 5:00 am) as fixed effects and subjects as random effects. Bonferroni post hoc analyses were performed when the main effects (group or times) or the interaction effect (group $\times$ times) were significant. The $p$ value of $<0.05$ was considered statistically significant. We determined if the slope of each subject's cortisol curve changed from the normal to the sleep deprived night by using a matched paired $t$-test to determine significance.

\section{RESULTS}

Table $\mathbf{1}$ shows demographic information for both groups; no significant differences were found across groups for any of the variables (Fishers's exact test $p>0.05$ ). There was no significant difference in age and BMI between patients and controls (Table 2). Fig. (1) shows the histograms for the latency to fall asleep after all night sleep deprivation. Controls had a mean latency to fall asleep of $3.3 \mathrm{~min}$ [sd 3.4] while the latencies of patients were significantly longer $[11.8 \mathrm{~min}$ (sd 12.3); $p<0.04]$. The patient data fell into two groups those overlapping with the controls and a subsample of 5 patients whose latencies were outside of the range of the normals - specifically, $12.5 \mathrm{~min}, 15.5 \mathrm{~min}, 24 \mathrm{~min}, 41 \mathrm{~min}$ and $54 \mathrm{~min}$. There was no significant effect of age on sleep latency. Neither CFS severity nor presence of comorbid fibromyalgia was predictive of either group (Table 2).

Table 1. Demographic Information

\begin{tabular}{|c|c|c|}
\hline & Control & CFS \\
\hline $\mathrm{N}$ & 12 & 15 \\
\hline \multicolumn{3}{|l|}{ Education level } \\
\hline High school or below & 2 & 3 \\
\hline Associate's degree or higher & 10 & 12 \\
\hline \multicolumn{3}{|l|}{ Marital status } \\
\hline Divorced & 2 & 5 \\
\hline Married & 3 & 6 \\
\hline Single & 7 & 4 \\
\hline \multicolumn{3}{|l|}{ Menstrual status } \\
\hline Menstruating & 9 & 9 \\
\hline Non-menstruating & 3 & 6 \\
\hline
\end{tabular}


Table 2. Sleep Characteristics for Controls, all Patients, and Patient Subgroups

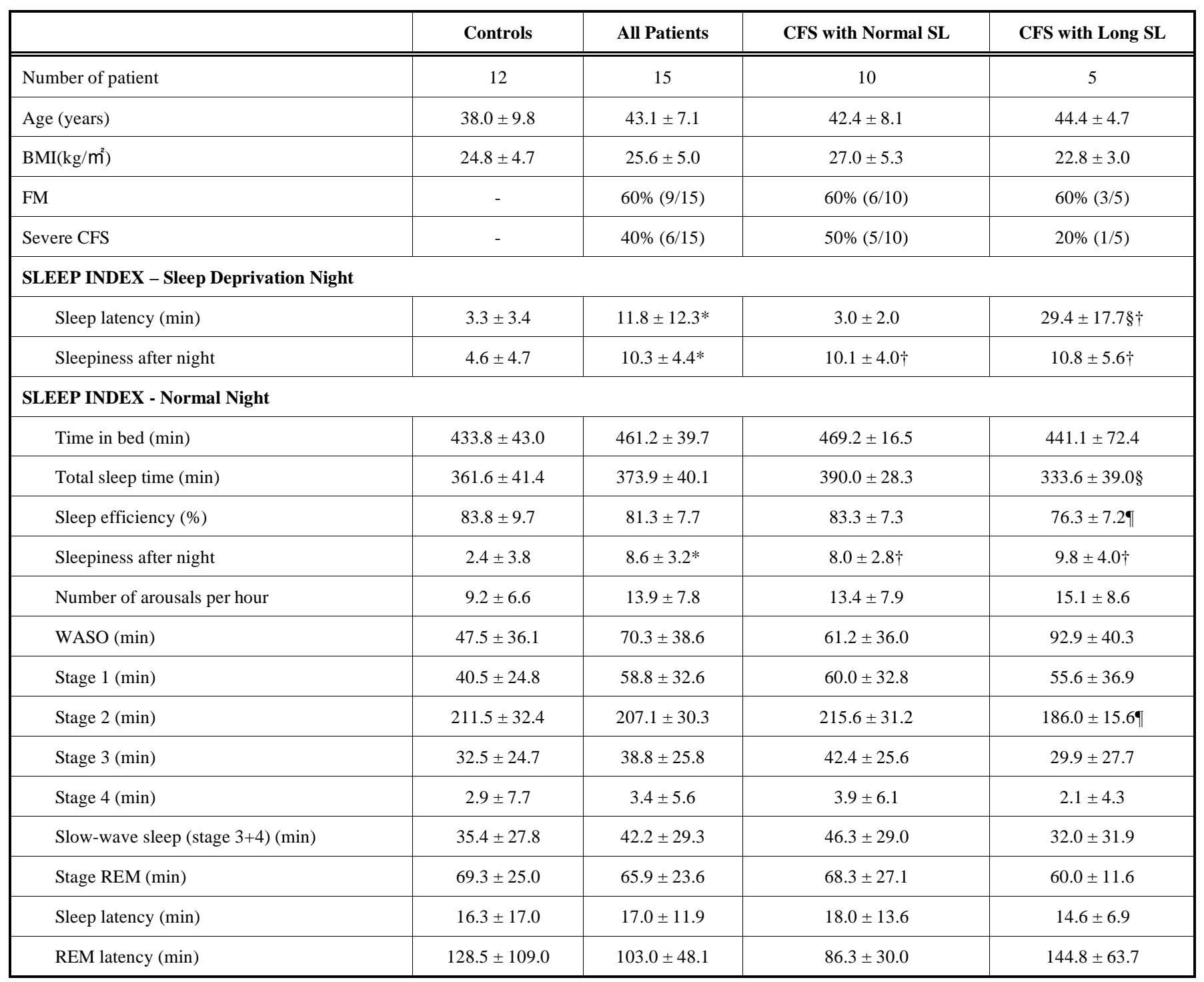

Values are mean \pm SD. SL: Sleep latency, WASO: wake after sleep onset.

*indicates significant difference between Control and CFS groups $(t$-test, $p<0.05)$. $\dagger$ indicates significant difference from Control (ANOVA, $p<0.05)$ ). $\S$ indicates significant difference between CFS subgroups (ANOVA, $p<0.05$ ). Iindicates significant difference between CFS subgroups (on tailed $U$-test, $p<0.05$ ).

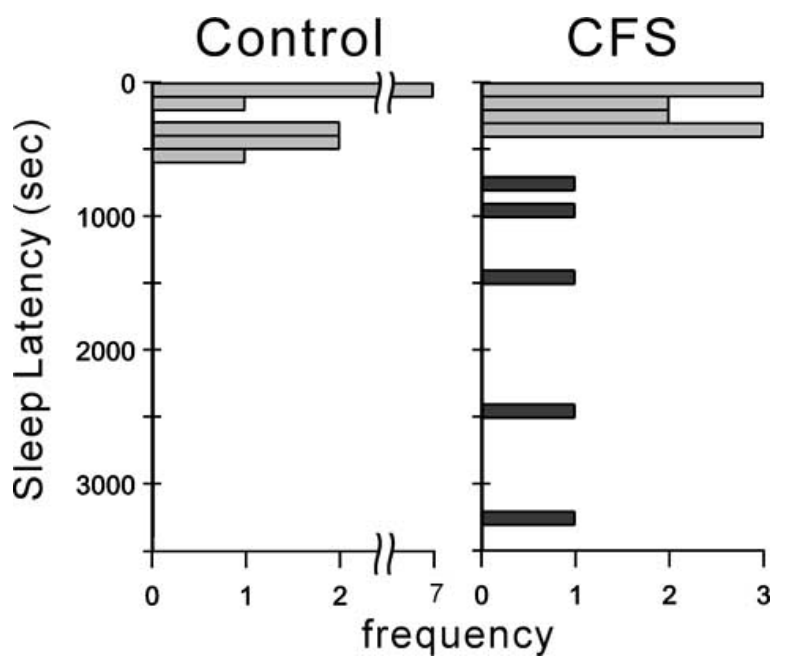

Fig. (1). The histograms of sleep latency after all night sleep deprivation for Control (Left) and CFS (Right).
We found several correlations for patients among sleep variables collected during the normal night of sleep and the sleep latency data following all night sleep deprivation. Specifically, inverse correlations were found between total sleep time and sleep efficiency on the normal night and sleep latency following sleep deprivation $(r=-0.59$ and $-0.58, p$ 's $<$ $0.03)$; positive correlations with sleep latency following sleep deprivation were found for latency to REM sleep $(\mathrm{r}=$ $0.79)$, time awake $(\mathrm{r}=0.57)$ and time awake as a percentage of all sleep related events $(0.61$; all $p$ 's $<0.03)$. There was no significant correlation between sleep latencies on the normal night and the sleep deprivation night.

There was no significant difference in sleep indices between Control and CFS groups, however patients with long sleep latencies after sleep deprivation had poorer sleep efficiencies (one tailed $U$-test; $p=0.053$ ) and shorter durations of Stage 2 and Total sleep time (one tailed $U$-test; $p=0.038$, ANOVA; $p<0.05)$ on their normal night of sleep than pa- 


\section{(a) Normal Night}

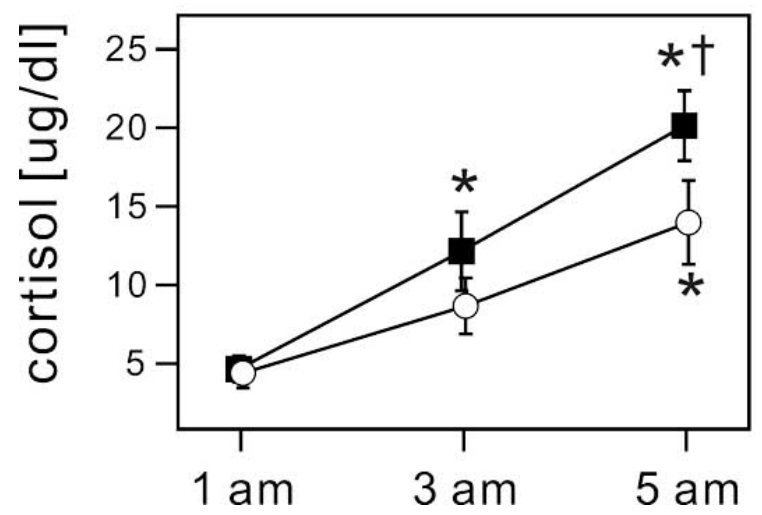

(b) Sleep Deprivation

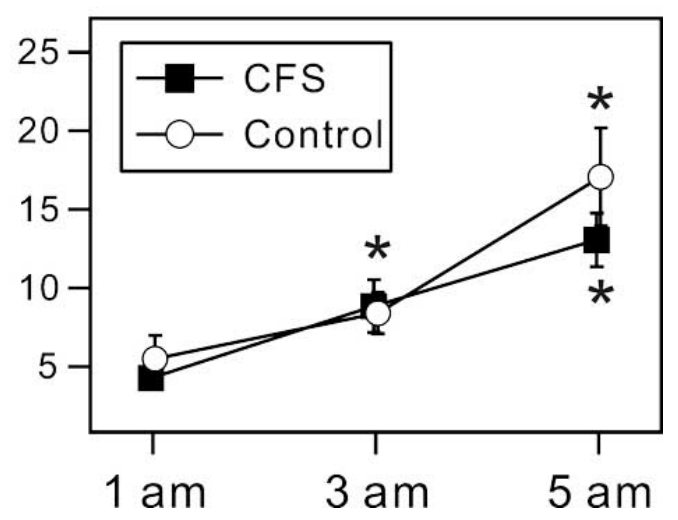

Fig. (2). The group averaged cortisol levels for CFS and Control. (a) normal sleep night, (b) sleep deprivation night. * indicates significant change from baseline (cortisol levels at 5:00 am). †indicates significant difference from Control.

tients with normal sleep latencies after sleep deprivation (see Table 2). Though, as expected, CFS patients as a group were significantly sleepier than Controls, there was no significant difference between the two CFS subgroups in the number reporting increased sleepiness after a night's sleep. This lack of effect may have reflected the small sample size of available data: 3 of 4 patients in the long latency group compared to 4 of 10 in the normal latency group reported being sleepier on the morning after their night of sleep than before it. Both CFS groups had similar rates of FM and similar rates of having severe CFS (see Table 2).

Fig. (2) shows the distributions of the cortisol data over time for CFS and Control groups. There was no significant group difference in cortisol levels at 1:00 am - either within or across study days. Both groups showed the expected increase across the night which is characteristic of the hormone's circadian pattern. Cortisol values did not vary across groups on either the normal or the sleep deprived nights (mixed model; $p>0.05$; see Fig. (2)), although post hoc testing did reveal a small difference at 5:00 am on the normal night with patients tending to be higher than controls $(p=0.03)$. However, a comparison of the slopes of the curves between normal and sleep deprived nights revealed the slope of the curve on the normal sleep night to be shifted up significantly for the CFS group only (paired $t$-test; $p<0.05$; compare panels $\mathrm{a}$ and $\mathrm{b}$ for $\mathrm{CFS}$ ).

\section{DISCUSSION}

As the time since awakening from sleep increases, sleep latency decreases [15], and one early study of young adults reported an average sleep latency of $30 \mathrm{sec}$ after a night of sleep deprivation [16]. After such a night of sleep deprivation in our laboratory, healthy subjects tended to fall asleep within 5 minutes, however 3 subjects took longer - falling asleep within 9 minutes. The somewhat longer sleep latencies manifested by our healthy subjects may reflect their older age. The CFS patients as a group showed a significantly longer latency to fall asleep after sleep deprivation, but the study population fell out into two groups with the largest group of 10 patients falling asleep within 5 minutes. However, the remaining 5 patients remained awake for a longer period than any control - suggesting that they may have a disorder of arousal. This outcome supports our hy- pothesis that some CFS patients may have a disorder of arousal which interferes with normal sleep and may, at least in part, be responsible for their disabling fatigue.

An alternative explanation has to do with CFS patients having an abnormality in sleep regulation, conceptualized some time ago as a two process model [17]. The first of these processes, basic sleep homeostasis or Process S, is thought to accumulate in wakefulness and can be operationally assessed by the amount and location of NREM delta sleep in the sleep cycle. The model has been supported by data showing increases in delta sleep in response to acute sleep deprivation [18]. Germane to this study is a recent report that CFS patients show a blunted initial delta sleep response to a 4 hour sleep delay - pointing to dysregulation of normal sleep regulatory processes [19]. That study suggests that basic sleep drive could be reduced in CFS and as a result, sleep latency could be prolonged even after sleep deprivation. Such an explanation does not require hyperarousal but rather a failure to down-regulate at sleep onset. However, to examine these two possibilities rigorously requires further study - specifically doing power spectral analysis of EEG recordings following a night of total sleep deprivation.

An important question is how to recognize patients falling into this subgroup. One way might be to evaluate sleep efficiency during a night of polysomnography. Our data suggest that patients with sleep efficiencies of about $75 \%$ on a night in the sleep lab would be at risk of falling into this category. However, sleep studies are expensive and so an alternative might be to have patients voluntarily deprive themselves of sleep and then go to the sleep lab for determination of their subsequent sleep latency. Such an approach could be used in future research seeking to understand the characteristics of this subgroup of CFS patients.

Patients with insomnia are thought to have a disorder of arousal, and thus it is possible that the unrefreshing sleep reported by patients with CFS with its resulting fatigue is simply a form of insomnia. Against that interpretation, however, are data from 12 patients with insomnia who reported having a chronic sleep problem lasting at least a year in which they either could not fall asleep in less then 45 minutes or were awake for at least an hour after falling asleep for at least four nights each week [20]. After an all night sleep 
deprivation, these patients had an average latency to fall asleep on the next morning of 3.8 minutes (sd 5.2). This value was quite similar to that shown by healthy controls in this study.

Surprisingly, in view of the fact that sleep is so frequently affected in CFS, no study, to our knowledge, has looked at cortisol and actual sleep architecture in CFS. A perfect control for these studies is a night of sleep deprivation where factors related to sleep do not come into play. In that condition, we found no difference between patients and controls. This result is consistent with earlier work [21]. Of interest, however, was a statistically significant shift upward of the slope of cortisol values over time during the normal night of sleep compared to that on the night of sleep deprivation plus a tendency for cortisol at $5 \mathrm{am}$ to be higher in patients than in controls on the normal night. Based on the well known fact that the adrenal cortex is activated by stress [7], we believe the data indicate stress due to disturbed sleep.

Our data should be viewed in the context of earlier studies of plasma cortisol in CFS in which blood has been periodically sampled throughout of night of normal sleep; these have reported disparate results. Two studies of plasma cortisol levels were done throughout the nighttime [22, 23]. Both reported no significant difference between patients and controls. However, in the early morning of the Di Giorgio study, patients tended to have higher cortisol values than controls. While such increased levels could reflect the stress induced by the disturbed sleep so commonly reported in CFS, an alternative interpretation is that our result could reflect a Type 1 statistical error.

This study had several limitations related to study population. First we studied women only, but we must point out that CFS is predominantly a problem in women's health in that the majority of patients are women [24]. Another limitation related to the relatively small number of subjects volunteering to be in it. While we did find that a third of our patient volunteers required rather long periods of time to fall asleep after a night of sleep deprivation, the total sample studied was quite small and having to do subgroup analyses makes samples even smaller. One issue limiting participation in this study was concerns about the consequences of sleep deprivation. Patients were afraid that participating in this study might make them sicker subsequently, and controls were worried about the consequences of sleep deprivation on their usual daily activities after the experiment. We found no evidence to support the patients' concern. Moreover, their participating in the study was important in that some of them were shown to have a marked delay in their ability to initiate sleep. This result suggests that this subgroup of patients may have a disorder of arousal that does not permit normal sleep. Further research is needed to better understand the causes of this problem in order to come up with treatments.

\section{ACKNOWLEDGEMENTS}

This work was supported by NIH \#AI-54478. The authors thank Dr. Roberta Moldow for doing the cortisol assays.

\section{REFERENCES}

Brimacombe M, Helmer D, Natelson BH. Clinical differences exist between patients fulfilling the 1988 and 1994 case definitions of chronic fatigue syndrome. J Clin Psychol Med Settings 2002; 9: 309-14.

[2] Krupp LB, Jandorf L, Coyle PK, Mendelson WB. Sleep disturbance in chronic fatigue syndrome. J Psychosom Res 1993; 37: 325-31.

[3] Sharpley A, Clements A, Hawton K, Sharpe M. Do patients with "pure" chronic fatigue syndrome (neurasthenia) have abnormal sleep? Psychosom Med 1997; 59: 592-6.

[4] Vassallo CM, Feldman E, Peto T, Castell L, Sharpley AL, Cowen PJ. Decreased tryptophan availability but normal post-synaptic 5$\mathrm{HT}_{2 \mathrm{c}}$ receptor sensitivity in chronic fatigue syndrome. Psychol Med 2001; 31: 585-91.

[5] Armitage R, Landis C, Hoffmann R, et al. Power spectral analysis of sleep EEG in twins discordant for chronic fatigue syndrome. $\mathrm{J}$ Psychosom Res 2009; 66: 51-57.

[6] Togo F, Natelson BH, Cherniack NS, Fitzgibbons J, Garcon C, Rapoport DM. Sleep structure and sleepiness in chronic fatigue syndrome with or without coexisting fibromyalgia. Arthritis Res Ther 2008; 10: R56.

[7] Natelson BH, Creighton D, McCarty R, Tapp WN, Pitman DL, Ottenweller JE. Adrenal indices of stress in laboratory rats. Physiol Behav 1987; 39: 117-25.

[8] Fukuda K, Straus SE, Hickie I, et al. The chronic fatigue syndrome: A comprehensive approach to its definition and study. Ann Intern Med 1994; 121: 953-9.

[9] Natelson BH, Johnson SK, DeLuca J, et al. Reducing heterogeneity in chronic fatigue syndrome: A comparison with depression and multiple sclerosis. Clin Infect Dis 1995; 21: 1204-10.

[10] Wolfe F, Smythe HA, Yunus MB, et al. The american college of rheumatology 1990 criteria for the classification of fibromyalgia. report of the multicenter criteria committee. Arthritis Rheum 1990; 33: $160-72$.

[11] Robbins LN, Cottler LB, Bucholz KK, Compton WM, North CS, Rourke KM. Diagnostic Interview Schedule for the DSM-IV (DISIV). St Louis, MO: University of Washington School of Medicine; 2000 .

[12] Moeller FG, Gillin JC, Irwin M, Golshan S, Kripke DF, Schuckit M. A comparison of sleep EEGs in patients with primary major depression and major depressoin secondary to alcoholism. J Affec Dis 1993; 27: 39-42.

[13] Togo F, Natelson BH, Cherniack NS, Klapholz M, Rapoport DM, Cook DB. Sleep is not disrupted by exercise in patients with chronic fatigue syndrome. Med Sci Sports Exercise 2010; 42(1): $16-22$.

[14] Rechtshaffen A, Kales A. A manual of standardized terminology, techniques and scoring system for sleep stages of human subjects. Los Angeles: Brain Information/ Brain Research Institute; 1968.

[15] Devoto A, Lucidi F, Violani C, Bertini M. Effects of different sleep reductions on daytime sleepiness. Sleep 1999; 22: 336-43.

[16] Carskadon MA, Dement WC. Effects of total sleep loss on sleep tendency. Percept Mot Skills 1979; 48: 495-506.

[17] Borbély AA. A two-process model of sleep regulatoin. Hum Neurobiol 1982; 1: 195-204.

[18] Borbély AA, Baumann F, Brandeis D, Strauch I, Lehmann D. Sleep deprivation: effect on sleep stages and EEG power density in man. Clin Neurophysiol 1981; 51: 483-93.

[19] Armitage R, Landis C, Hoffmann R, et al. The impact of a 4-hour sleep delay on slow wave activity in twins discordant for chronic fatigue syndrome. Sleep 2007; 30: 657-62.

[20] Bonnet MH Arand DL. Activity, arousal, and the MSLT in patients with insomnia. Sleep 2000; 23: 205-12.

[21] Cleare AJ. The neuroendocrinology of Chronic Fatigue Syndrome. Endocr Rev 2003; 24: 236-52.

[22] Crofford LJ, Young EA, Engleberg NC, et al. Basal circadian and pulsatile ACTH and cortisol secretion in patients with fibromyalgia and/or chronic fatigue syndrome. Brain Behav Immun 2004; 18: 314-25. 
[23] Di Giorgio A, Hudson M, Jerjes W, Cleare AJ. 24-hour pituitary and adrenal hormone profiles in chronic fatigue syndrome. Psychosom Med 2005; 67: 433-40.
[24] Jason LA, Richman JA, Rademaker AW, et al. A community-based study of chronic fatigue syndrome. Arch. Intern. Med 1999; 159: 2129-2137.

(C) Nakamura et al.; Licensee Bentham Open.

This is an open access article licensed under the terms of the Creative Commons Attribution Non-Commercial License (http://creativecommons.org/licenses/by-nc/3.0/) which permits unrestricted, non-commercial use, distribution and reproduction in any medium, provided the work is properly cited. 